Thermal-infrared adaptive optics imaging- and spectro-polarimetry with the Infrared Camera and Spectrograph (IRCS) for the Subaru Telescope

Hiroshi Terada, Mitsuhiko Honda, Takashi Hattori, Tomoyuki Kudo, Jun Hashimoto, et al.

Hiroshi Terada, Mitsuhiko Honda, Takashi Hattori, Tomoyuki Kudo, Jun Hashimoto, Makoto Watanabe, "Thermal-infrared adaptive optics imagingand spectro-polarimetry with the Infrared Camera and Spectrograph (IRCS) for the Subaru Telescope," Proc. SPIE 10702, Ground-based and Airborne Instrumentation for Astronomy VII, 107022Y (6 July 2018); doi: $10.1117 / 12.2313303$ 


\title{
Thermal Infrared Adaptive Optics Imaging- and Spectro-Polarimetry with the Infrared Camera and Spectrograph (IRCS) for the Subaru Telescope
}

\author{
Hiroshi Terada ${ }^{\mathrm{a}}$, Mitsuhiko Honda ${ }^{\mathrm{b}}$, Takashi Hattori ${ }^{\mathrm{c}}$, Tomoyuki Kudo ${ }^{\mathrm{c}}$, Jun Hashimoto ${ }^{\mathrm{d}}$, and \\ Makoto Watanabe ${ }^{\mathrm{e}}$ \\ aThirty Meter Telescope Project, National Astronomical Observatory of Japan, 100 West \\ Walnut, Suite 300, Pasadena, CA 91124, USA \\ ${ }^{\mathrm{b}}$ Department of Physics, Kurume University of School of Medicine, 67 Asahi-machi, Kurume, \\ Fukuoka 830-0011, Japan \\ ${ }^{c}$ Subaru Telescope, National Astronomical Observatory of Japan, 650 North A'ohoku Place, \\ Hilo, HI 96720, USA \\ ${ }^{\mathrm{d}}$ AstroBiology Center, National Institutes of Natural Sciences, 2-21-1 Osawa, Mitaka, Tokyo \\ 181-8588, Japan \\ eDepartment of Applied Physics, Okayama University of Science, 1-1 Ridaicho, Kita-ku, \\ Okayama 700-0005, Japan
}

\begin{abstract}
A thermal-infrared polarimetric capability of the Infrared Camera and Spectrograph (IRCS) for the Subaru Telescope is described. A new half-wave retarder for the thermal-infrared band in $2-5 \mu \mathrm{m}$ is introduced into the Waveplate Unit of the Nasmyth platform on the infrared side of the telescope to realize imaging- and low resolution spectro-polarimetry in that wavelength region. Through day-time calibrations using a wire-grid polarizer, the peak efficiency of the polarization is found to be $90-98 \%$ consistently in both imaging- and spectropolarimetry in the thermal-infrared bands. In 2016 May and 2017 June, two engineering observing runs have been carried out to verify the on-sky performance.
\end{abstract}

Keywords: polarimetry, adaptive optics, thermal infrared, imaging, spectroscopy, dust, protoplanetary disk, polarimetric differential technique

\section{INTRODUCTION}

Polarimetry in astronomy is a unique tool to prove various physical conditions of astronomical objects such as magnetic field, geometry of scattering circumstellar/circumnuclear materials, and dust alignment around the objects. Though it becomes more popular in astronomical telescopes, polarimetry is not yet a basic function like imaging and spectroscopy among the existing major facilities. In particular, the polarimetric capability in the infrared wavelengths is not implemented in most of the astronomical instruments and therefore polarimetric science has not been fully explored so far in the infrared bands where a high spatial resolution could be stably achieved by an adaptive optics technology at large $(8-10 \mathrm{~m})$ telescopes. Moreover, the thermal-infrared (TIR) polarimetry is so rare and observing opportunities are quite limited, although it is very powerful to characterize dust properties around a wide variety of astronomical objects.

Infrared Camera and Spectrograph (IRCS) is the first generation instrument at the Subaru Telescope ${ }^{1,2}$ and has been operating very stably for these two decades since the telescope first light year of $1999 .^{3,4}$ It was originally mounted at the Cassegrain focus in conjunction with the first generation of the adaptive optics system at the Subaru Telescope (AO36) ${ }^{5}$ and then moved to the Nasmyth platform on the infrared side (NsIR) of the

Further author information: (Send correspondence to H.T.)

H.T.: E-mail: terada@naoj.org, Telephone: 16263951670

Ground-based and Airborne Instrumentation for Astronomy VII, edited by Christopher J. Evans, Luc Simard, Hideki Takami, Proc. of SPIE Vol. 10702, 107022Y · (c) 2018 SPIE · CCC code: 0277-786X/18/\$18 · doi: 10.1117/12.2313303 


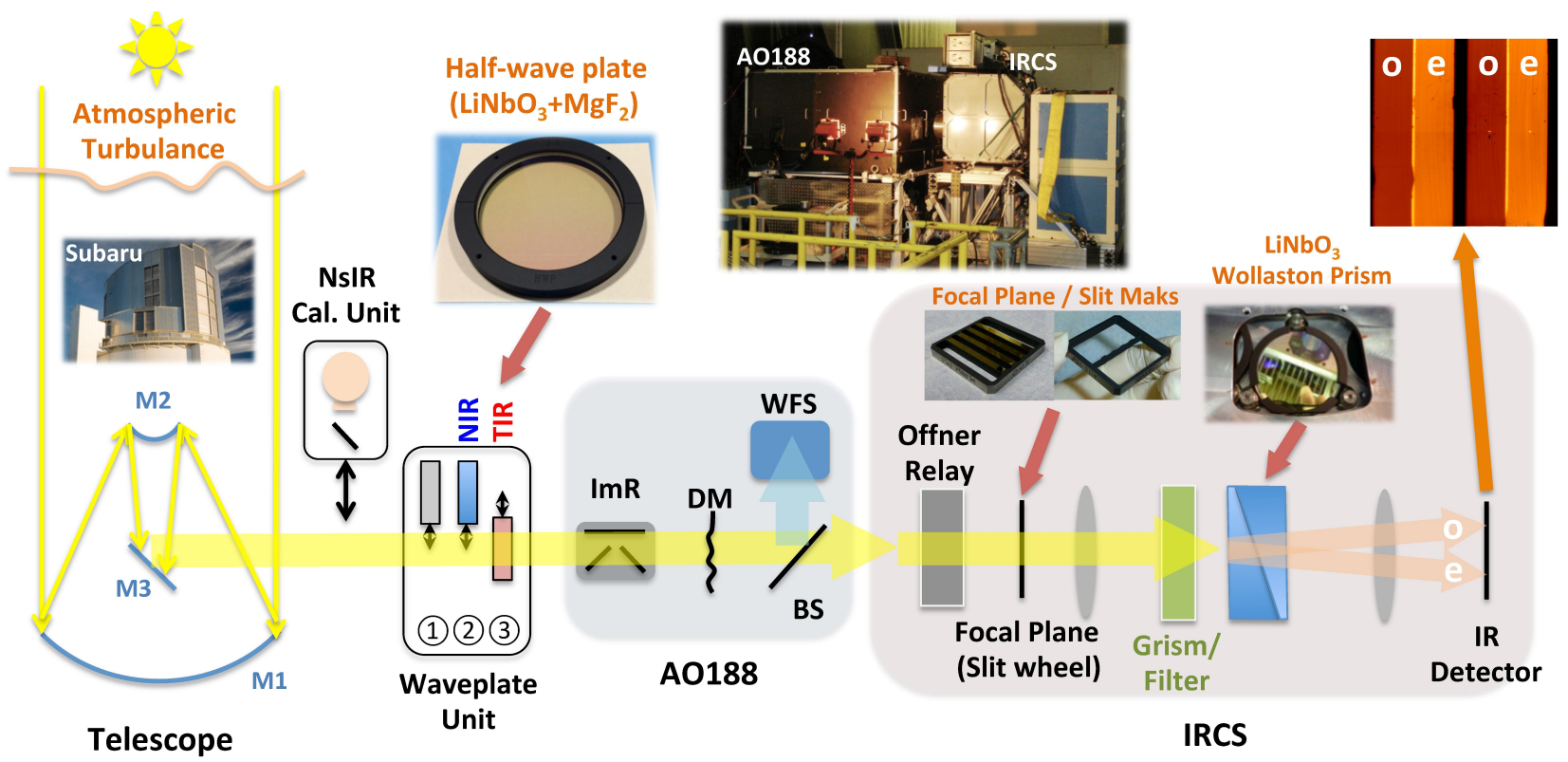

Figure 1. Optical layout of the IRCS polarimetry configuration at the NsIR of the Subaru Telescope . Light coming from the sky goes through the telescope primary (M1), secondary (M2), and tertiary mirrors (M3) to be directed toward the NsIR of the telescope.

telescope in 2005 to combine with the second generation of the laser-guide star AO system (AO188). ${ }^{6}$ Infrared polarimetric function ${ }^{7}$ was introduced in 2013 into the instrument by using a Wollaston prism which is made of $\mathrm{LiNbO}_{3}$ as a polarizer. Wavelength coverage of this infrared polarimetry is limited to near-infrared (NIR) band $(0.95-2.5 \mu \mathrm{m})$ due to no sufficient transmittance of the original retarder (Quartz $+\mathrm{MgF}_{2}$ half-wave plate) at the NsIR focus of the telescope.

\section{OPTICAL LAYOUT AND SPECIFICATION}

The optical layout for the TIR polarimetry configuration of the IRCS+AO188 is shown in Fig. 1.

Following a successful commissioning of the NIR imaging- and spectro-polarimetry through engineering runs from 2013 through 2015, we expanded the polarimetric capability of the IRCS+AO188 into the TIR wavelengths up to $5 \mu \mathrm{m}$ with a new $\mathrm{LiNbO}_{3}+\mathrm{MgF}_{2}$ half-wave plate as a retarder. The new wave plate was manufactured by Kogakugiken Corp. (Kanagawa, Japan), which has a sufficient throughtput ( $>90 \%$ ) throughout $2-5 \mu \mathrm{m}$ and a clear aperture of $100 \mathrm{~mm}$ in diameter. We installed it in the third slot of the Waveplate Unit in 2016 May which is located at the closest to the instrument and the field-of-view with the clear aperture is secured to be $\phi 68^{\prime \prime}$. The Wollaston prism in the dispersion wheel and focal plane/slit masks in the slit wheel which have been utilized for the NIR polarimetry are available also for the TIR bands, because those are designed for accommodation to the full wavelength coverage $(0.95-5.0 \mu \mathrm{m})$ of the IRCS+AO188. For imaging-polarimetry, only the fine pixel scale (20mas/pixel) camera is used for the TIR bands due to its high background from the warm optics. On the other hand, only the $52 \mathrm{mas} /$ pixel camera is available for spectro-polarimetry and slit widths are chosen from $0^{\prime \prime} .6,0^{\prime \prime} .15,0^{\prime \prime} .1$, and $0^{\prime \prime} .22$. A lateral chromatism of the Wollaston prism causes an overlap of the field apertures in $L$ and $M^{\prime}$ as seen in Fig. 2. As a result, the field of view for imaging-polarimetry at $L^{\prime}$ and $M^{\prime}$ consists of two apertures with an area of $4.0^{\prime \prime}$ and $3.0^{\prime \prime}$ respectively and the slit length for $L$ spectro-polarimetry is limited up to $4.0^{\prime \prime}$. The available mode for the TIR polarimetry of the IRCS+AO188 is summarized in Table 1.

\section{POLARIMETRIC CALIBRATION}

In general, the polarimetric calibration is much harder at a Nasmyth platform than the one at a Cassegrain focus, because a tertiary mirror produces an instrumental polarization. ${ }^{8,9}$ It is estimated to be at a level of $\mathrm{P} \sim 0.5 \%$ 

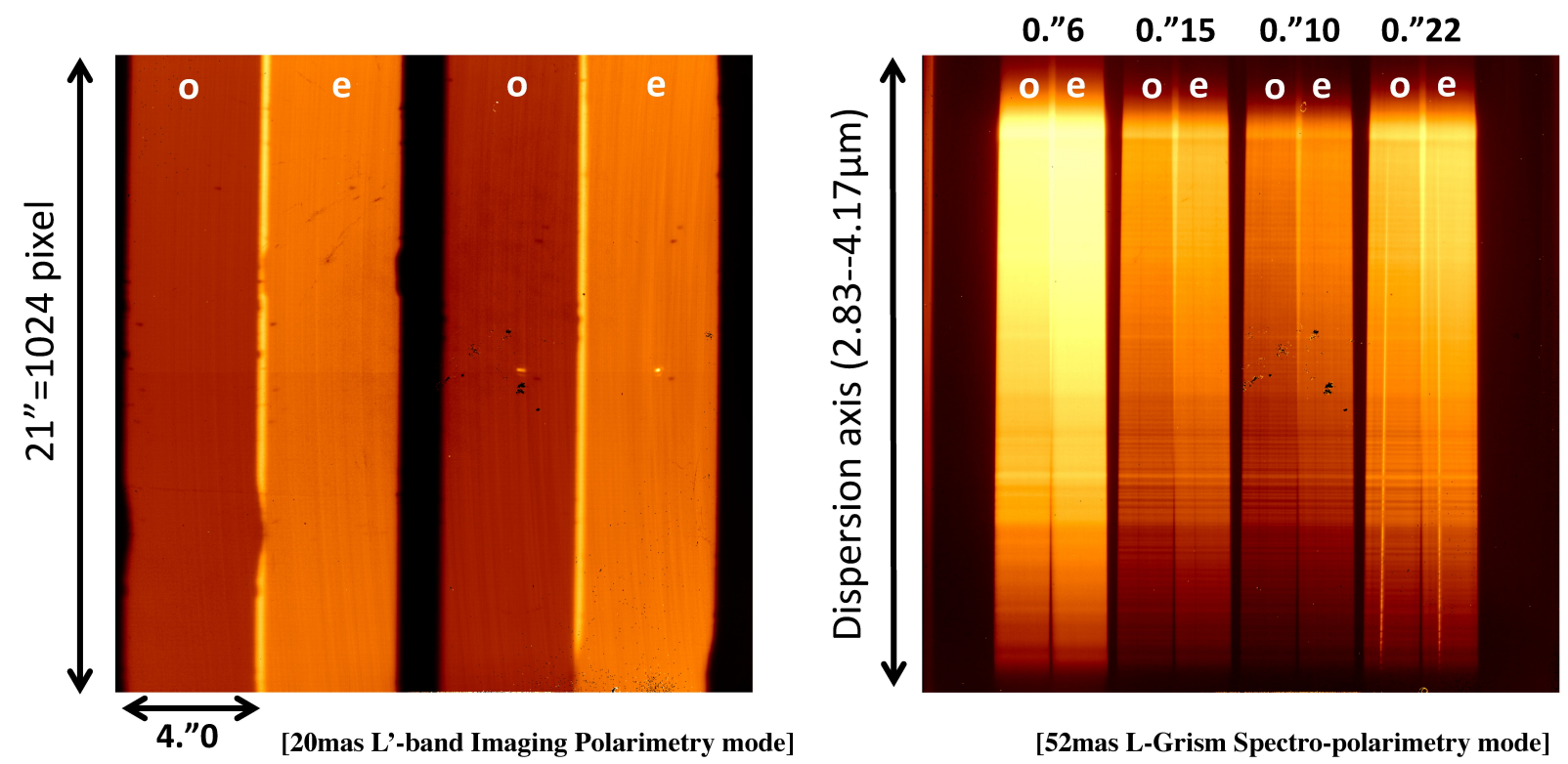

Figure 2. Polarimetric image at $L^{\prime}$ and polarimetric spectrum at $L$.

at the Subaru telescope in the wavelength region of $2-5 \mu \mathrm{m}$ and it will be problem in case of measurements for $<1 \%$ polarization. In addition to that complexity, an overcoating on the silver mirror surfaces of the AO188 Image Rotator (ImR) degrades polarization efficiency with a function of its rotating angle in this system.

In the day time calibration, we installed a wire-grid polarizer for $2-5 \mu \mathrm{m}$ with a clear aperture of $110 \mathrm{~mm}$ (manufactured by Polatechno Co. Ltd., Niigata, Japan) in the first slot of the Waveplate Unit to measure the polarization efficiency with a $5^{\circ}$ step of the $\mathrm{AO} 188 \mathrm{ImR}$ angle from $0^{\circ}$ to $90^{\circ}$ by using a halogen lamp as a light source installed at the NsIR focus of the telescope. Fig. 3 shows results of the measured polarization efficiency for both imaging and spectroscopy. As expected from theoretical calculation, the polarization efficiency becomes minimum around the AO ImR angle of $45^{\circ}$. The peak efficiency of the polarization is found to be $90-98 \%$. With an angle range of $0-10^{\circ}$ or $80-90^{\circ}$, the degradation of the polarization is kept to be $>80 \%$. This will limit a position angle of the observing field of view and the slit / field position angle is almost uniquely determined at a given hour angle of the observations.

A finer-step $\left(1^{\circ}\right)$ result of the polarization efficiency around the AO188 ImR angle of $0^{\circ}$ is shown in Fig. 4 . In order to obtain the polarization efficiency of $>90^{\circ}$, the AO188 ImR angle needs to be within a range of $-6^{\circ}$ to $4^{\circ}$.

Table 1. Summary of the IRCS+AO188 TIR polarimetric functions.

\begin{tabular}{|c|c|c|}
\hline \multicolumn{3}{|c|}{ Thermal Infrared (TIR) Polarimetry $(1.92-4.80 \mu \mathrm{m})$} \\
\hline Mode & Imaging Polarimetry & Spectro-Polarimetry \\
\hline Band & $\begin{array}{c}\text { Broad bands: } \mathrm{K}, \mathrm{K}^{\prime}, \mathrm{L}^{\prime}, \mathrm{M}^{\prime} \\
\text { Narrow bands in } 2-5 \mu \mathrm{m}\end{array}$ & $\begin{array}{l}\left.\text { Grism K (R } \sim 869 \lambda \mathrm{w} / 0.10^{\prime \prime} \text { slit }\right) \\
\left.\text { Grism L ( } \sim 269 \lambda \mathrm{w} / 0.10^{\prime \prime} \text { slit }\right)\end{array}$ \\
\hline Pixel scale & 20mas/pixel & $52 \mathrm{mas} /$ pixel \\
\hline $\begin{array}{l}\text { FoV of } \\
\text { mask/slit }\end{array}$ & $\begin{array}{l}\mathrm{K}, \mathrm{K}^{\prime}: 2 \text { Strips of } 4.4^{\prime \prime}(\mathrm{W}) \times 21^{\prime \prime}(\mathrm{L}) \\
\mathrm{L}^{\prime} 2 \text { Strips of } 4.0^{\prime \prime}(\mathrm{W}) \times 21^{\prime \prime}(\mathrm{L}) \\
\mathrm{M}^{\prime}: 2 \text { Strips of } 3.0^{\prime \prime}(\mathrm{W}) \times 21^{\prime \prime}(\mathrm{L})\end{array}$ & $\begin{array}{l}0.60^{\prime \prime}(\mathrm{W}) \times 4.0^{\prime \prime}(\mathrm{L}) \\
0.15^{\prime \prime}(\mathrm{W}) \times 4.0^{\prime \prime}(\mathrm{L}) \\
0.10^{\prime \prime}(\mathrm{W}) \times 4.0^{\prime \prime}(\mathrm{L}) \\
0.22^{\prime \prime}(\mathrm{W}) \times 4.0^{\prime \prime}(\mathrm{L})\end{array}$ \\
\hline
\end{tabular}



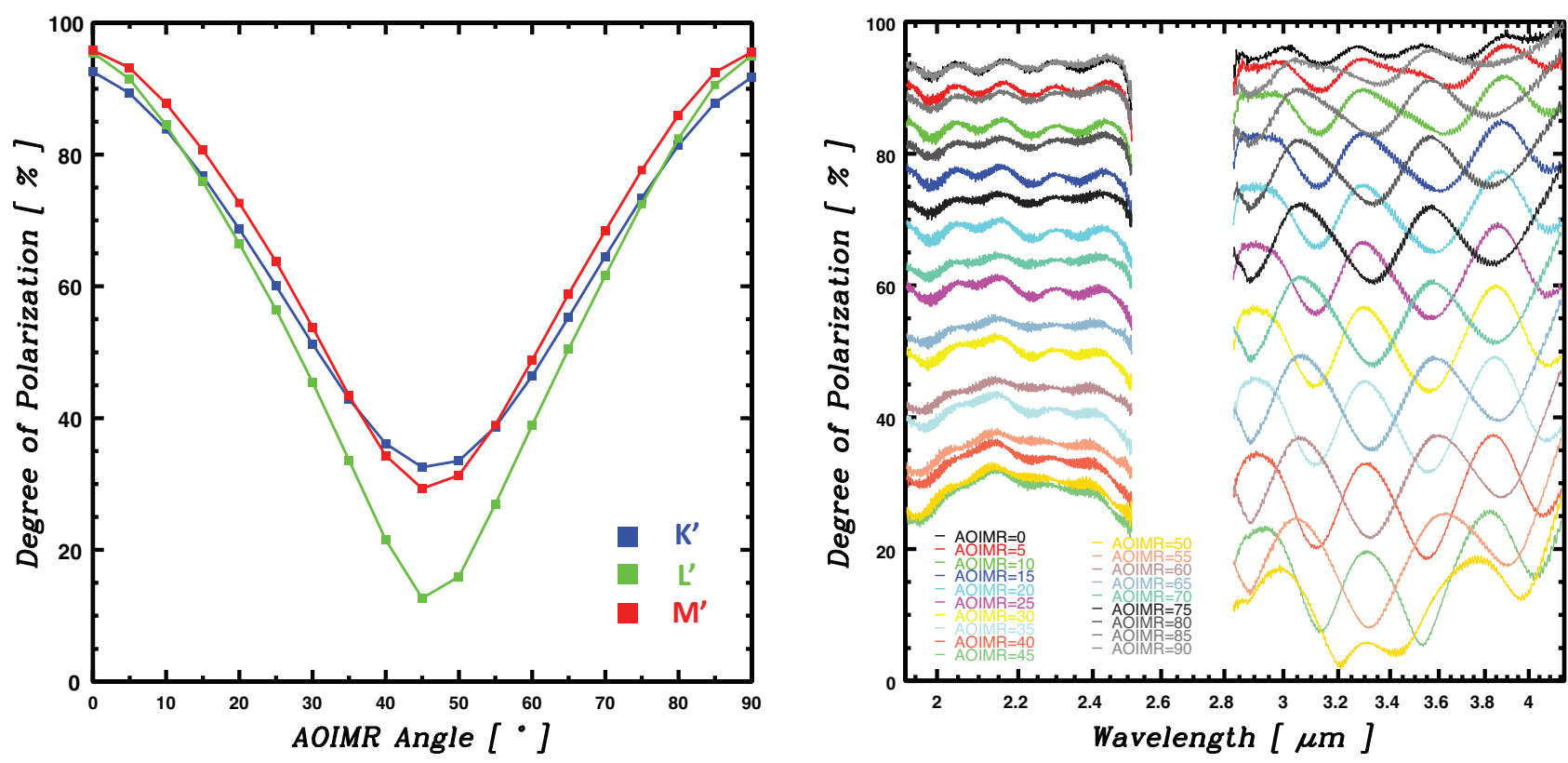

Figure 3. Polarization efficiency vs. AO188 ImR.

An efficiency curve interpolated from this calibration result can be applied to observing polarization correction.

\section{OBSERVING PERFORMANCE}

Two engineering observing runs were conducted in 2016 May and 2017 June to verify the on-sky performance of the TIR polarimetry mode of the IRCS+AO188. Throughput of the TIR half-wave plate is measured with FS 147 as $89.9 \%, 91.9 \%$, and $90.7 \%$ at $K^{\prime}, L^{\prime}$, and $M^{\prime}$, respectively. Increase of background at $L^{\prime}$ and $M^{\prime}$ is $17 \%$ and $21 \%$. Observing targets in the runs are several polarized standard stars, unpolarized standard stars, and also two Herbig Ae stars for the performance demonstration. Details of the observations are summarized in Table 2.

\subsection{Imaging Polarimetry}

Fig. 5 shows results of imaging polarimetry for the unpolarized and polarized stars with and without the instrumental polarization (IP) correction. As for the correction of the IP caused by the telescope M3 and the AO188, we apply the same method that has been used for the HiCIAO ${ }^{18}$ data.

The IP corrected data for the unpolarized stars exhibit no significant polarization (Fig. 5(a)), while the errors are somehow consistently larger than the ones without the IP correction. No IP corrected data are also well below $1 \%$ for all the unpolalrized stars. Regarding the polarized stars, there are a good correlation between measured and previously reported values as seen in Fig. 5(b). Detailed investigation for the best calibration analysis to remove the instrumental polarization is on-going.

Although a polarimetric differential imaging technique with dual-beam polarimeters has been well demonstrated (e.g., Ref. [19]), the IRCS+AO188 TIR imaging polarimetry suffers from the image elongation which is caused by a large lateral chromatism of the $\mathrm{LiNbO}_{3}$ Wollaston prism. We estimated contribution of the chromatism on the polarimetric image directly from the spectra in Fig. 6. At $L^{\prime}(3.42-4.12 \mu \mathrm{m})$, it results in 0." 22 . Even with a narrow band filter of $\mathrm{H}_{2} \mathrm{O}$ Ice $(2.974-3.126 \mu \mathrm{m})$, it is $0 .{ }^{\prime \prime} 035$ which damages a precise extraction of the differential signal. Manufacturability of alternative Wollaston prism made of a better material (such as AgGaS 2 ) with the equivalent size (D 35mm) to the current one is being explored for sufficiently low lateral chromatism. 


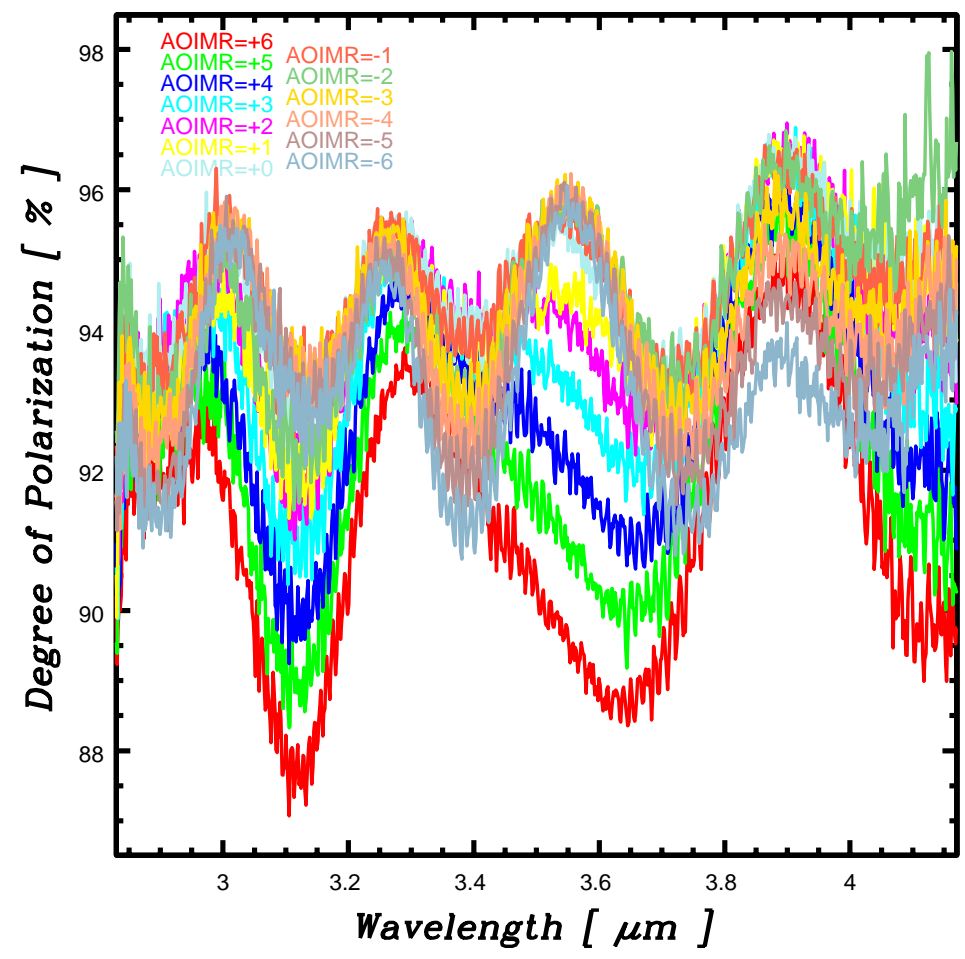

Figure 4. Details of polarization efficiency around $0^{\circ}$ for the AO188 ImR angle.

\subsection{Spectro-Polarimetry}

Polarimetric spectra in this mode are found to show a ripple feature which is supposed to originate from the new TIR half-wave plate. The same symptom is often seen in other polarimetric facilities (e.g., UIST at UKIRT ${ }^{20}$ ). Spectral binning by over 8-9 pixels which corresponds to $>0 . " 45$ slit width can smear out the feature (Fig. 7), but the resultant spectral resolution is decreased for the case of $0 .{ }^{\prime \prime} 10,0 .{ }^{\prime \prime} 15$, and $0 .{ }^{\prime \prime} 22$ slit use. We attempted to cancel the ripple feature with an A0V star (HR 2757) in an open use program of the telescope time by following a method described in Ref. [21], which is based on the operations of $\theta_{W P}=\left[0^{\circ}, 45^{\circ}, 22.5^{\circ}, 67.5^{\circ}, 90^{\circ}, 135^{\circ}, 112.5^{\circ}, 157.5^{\circ}\right]$. The result shows a successful cancel-out of the artificial feature (Fig. 8) and we are establishing the standard operational procedure with this method.

Polarimetric differential spectra shouldn't be affected by the large chromatism mentioned in the above. However, only 52mas/pixel camera is available for the low spectral resolution spectroscopy at $L$ and the polarimetric spectra are under-sampled. Thus, it may be difficult to go into a closer working angle also in this mode. The performance will be assessed through our on-going science observing program with the IRCS+AO188 to detect a water ice signature in scattered light from face-on protoplanetary disks.

\section{SUMMARY AND FUTURE PROSPECT}

The new function of the thermal infrared polarimetry function for the IRCS was described. While most of the optical components are shared with the near infrared polarimetry function of the same instrument, the waveplate dedicated to this new function was newly installed. Performance for the detection of the $\mathrm{P}<1 \%$ polarization was confirmed through the on-sky observing runs. Capability of polarimetric differential imaging is limited at this moment by a large lateral chromatic dispersion of the current material of $\mathrm{LiNbO}_{3}$ for the Wollaston prism and investigation is on-going to find availability of a new Wollaston prism with the proper size $(\sim 35 \mathrm{~mm})$ made of better material to produce smaller lateral chromatic dispersion. 


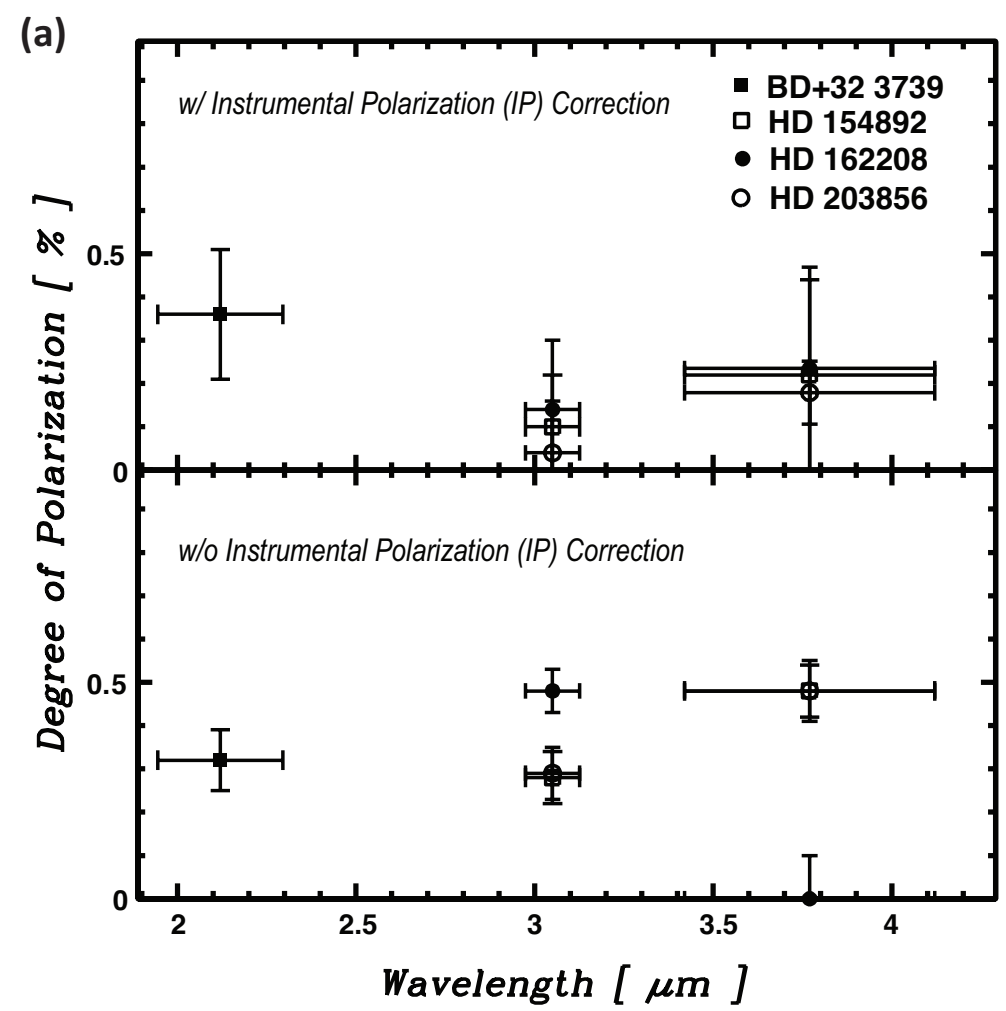

(b)
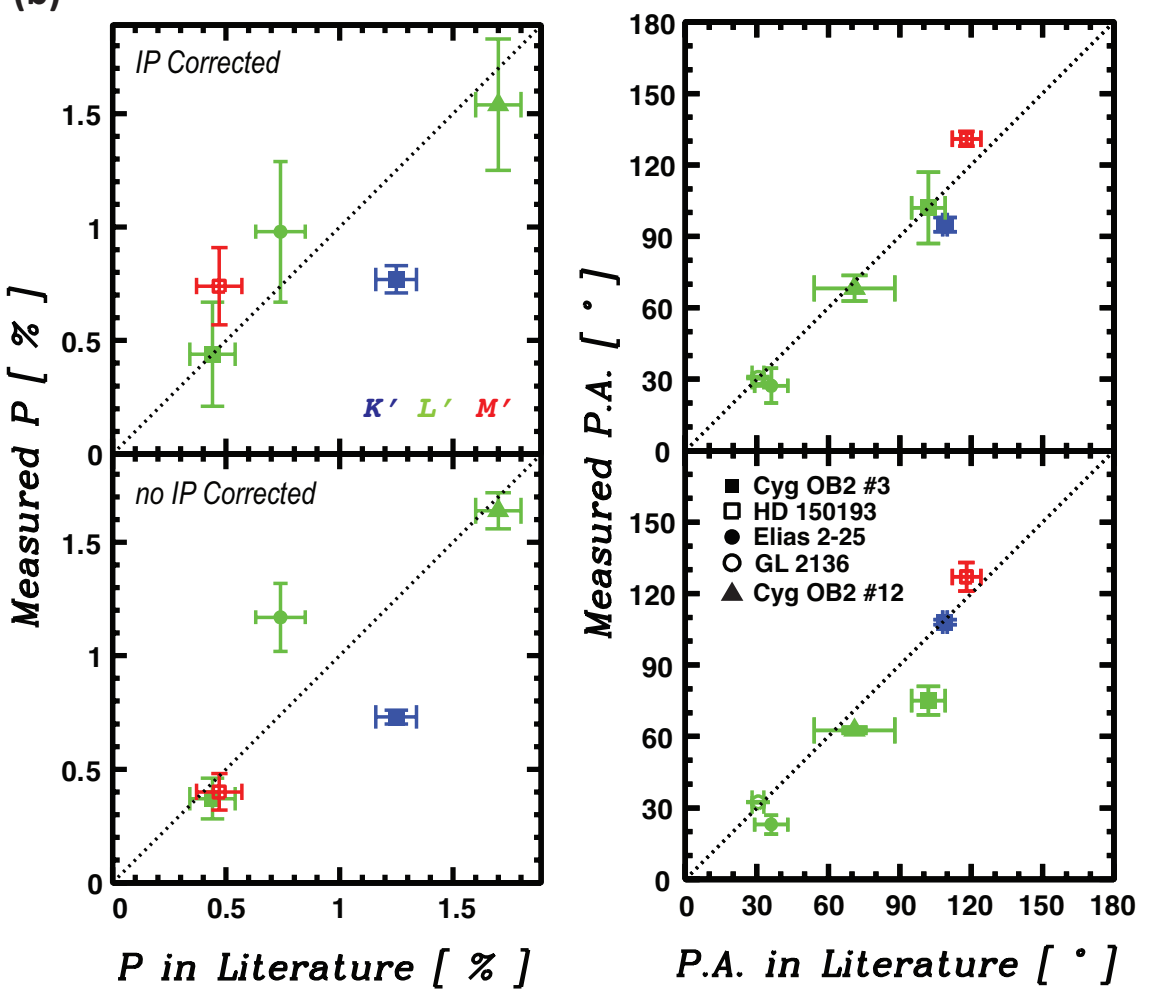

Figure 5. (a) Measurement of unpolarized standard stars. (b) Polarization degree and angle check with known polarized stars. 


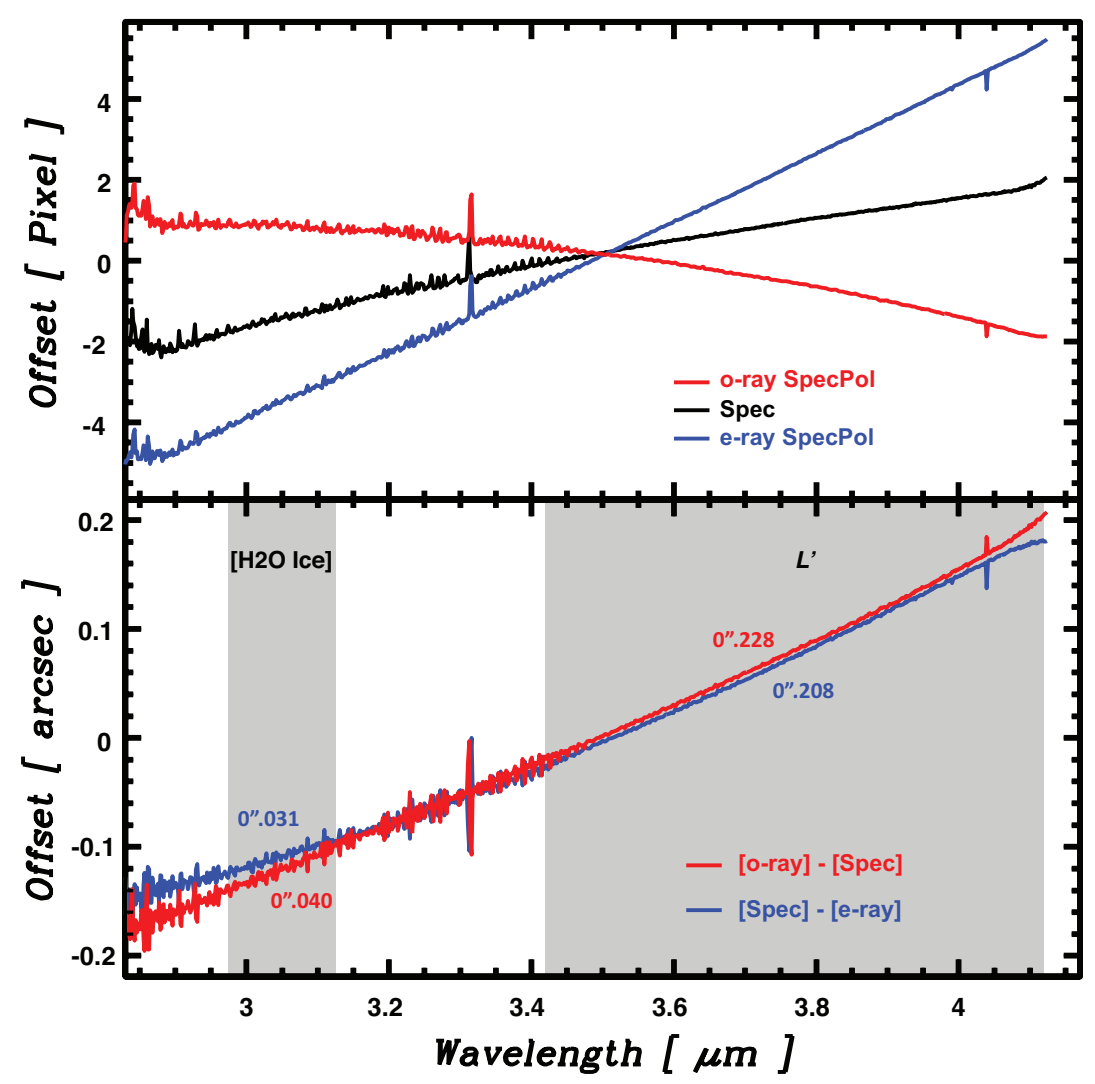

Figure 6. Lateral chromatism of $\mathrm{LiNbO}_{3}$ Wollaston prism. The upper panel shows a position of both polarimetric spectra (o-ray in red and e-ray in blue) and standard (non-polarimetric) spectrum (without the Wollaston prism) in black. The lower panel is a positional difference of the spectra which represents a lateral chromatism as a function of wavelength (at $L, 2.83-4.17 \mu \mathrm{m})$.

\section{ACKNOWLEDGMENTS}

We wish to thank the support of the science operations and instrument maintenance staff of the Subaru Telecope Project, in particular T.-S. Pyo, J. Bulger, J. H. Kim, M. Wung, and M. Weber. We also thank M. Tamura and the HiCIAO group for providing with the waveplate unit originally developed for the HiCIAO instrument at the Nasmyth focus of the Subaru Telescope.

\section{REFERENCES}

[1] A. T. Tokunaga, N. Kobayashi, J. Bell, G. K. Ching, K. Hodapp, J. L. Hora, D. Neil, P. M. Onaka, J. T. Rayner, L. Robertson, D. W. Warren, M. Wever, and T. T. Young, "Infrared Camera and Spectrograph for the Subaru Telescope", in Infrared Astronomical Instrumentation, A. M. Fowler, ed., Proc. SPIE, 3354, pp. 512-524, 1998.

[2] N. Kobayashi, A. T. Tokunaga, H. Terada, M. Goto, M. Weber, R. Potter, P. M. Onaka, G. K. Ching, T. T. Young, K. Fletcher, D. Neil, L. Robertson, D. Cook, M. Imanishi, and D. W. Warren, "IRCS: Infrared Camera and Spectrograph for the Subaru Telescope", in Optical and Infrared Instrumentation and Detectors, M. Iye and A. F. Moorwood, eds., Proc. SPIE, 4008, pp. 1056-1066, 2000.

[3] H. Terada, N. Kobayashi, A. T. Tokunaga, T. S. Pyo, M. Goto, M. Weber, R. Potter, and P. M. Onaka, "Infrared Camera and Spectrograph for the Subaru Telescope: performance improvement with the new Aladdin-III array", in Optical and Infrared Instrumentation and Detectors, M. Iye and A. F. Moorwood, eds., Proc. SPIE, 4841, pp. 1306-1314, 2003. 


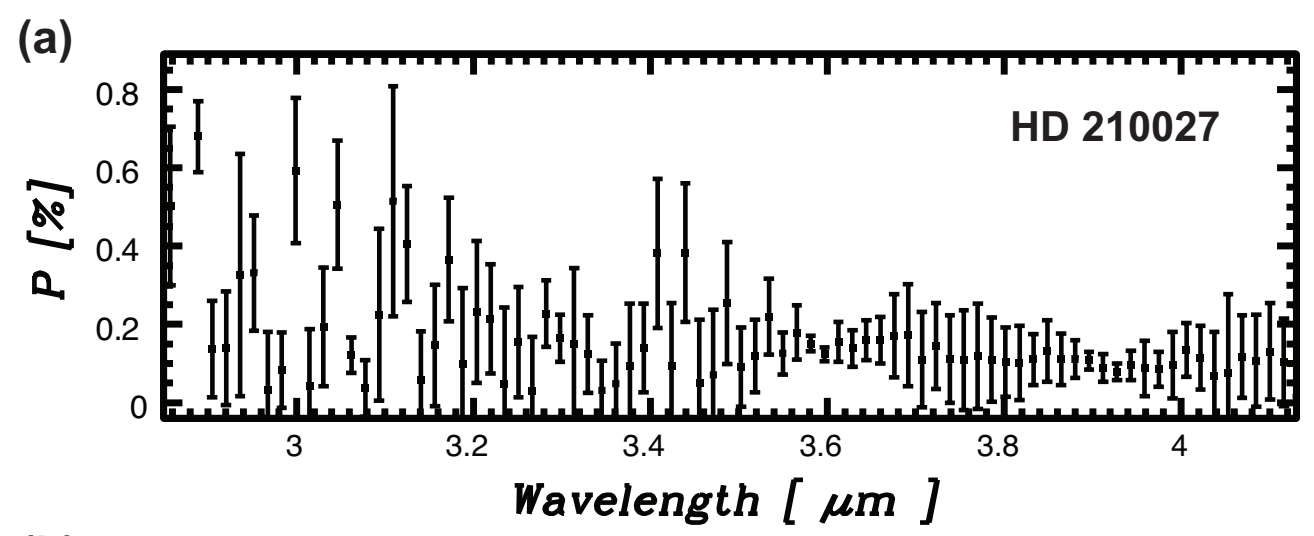

(b)

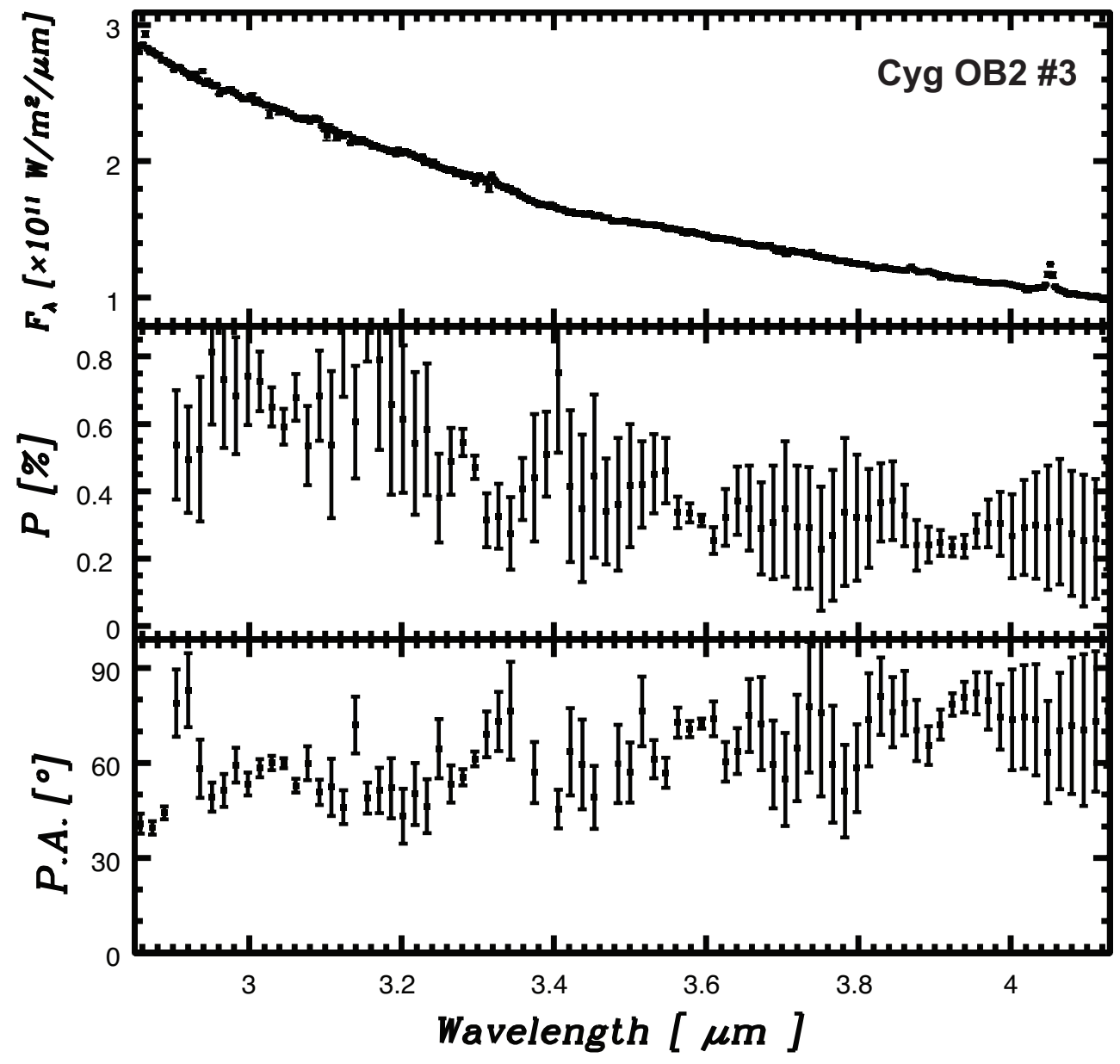

Figure 7. (a) Measurement of a unpolarized standard star, HD 210027. (b) Polarization degree and angle check with an interstellar polarimetric object, Cyg OB2 No. 3. 


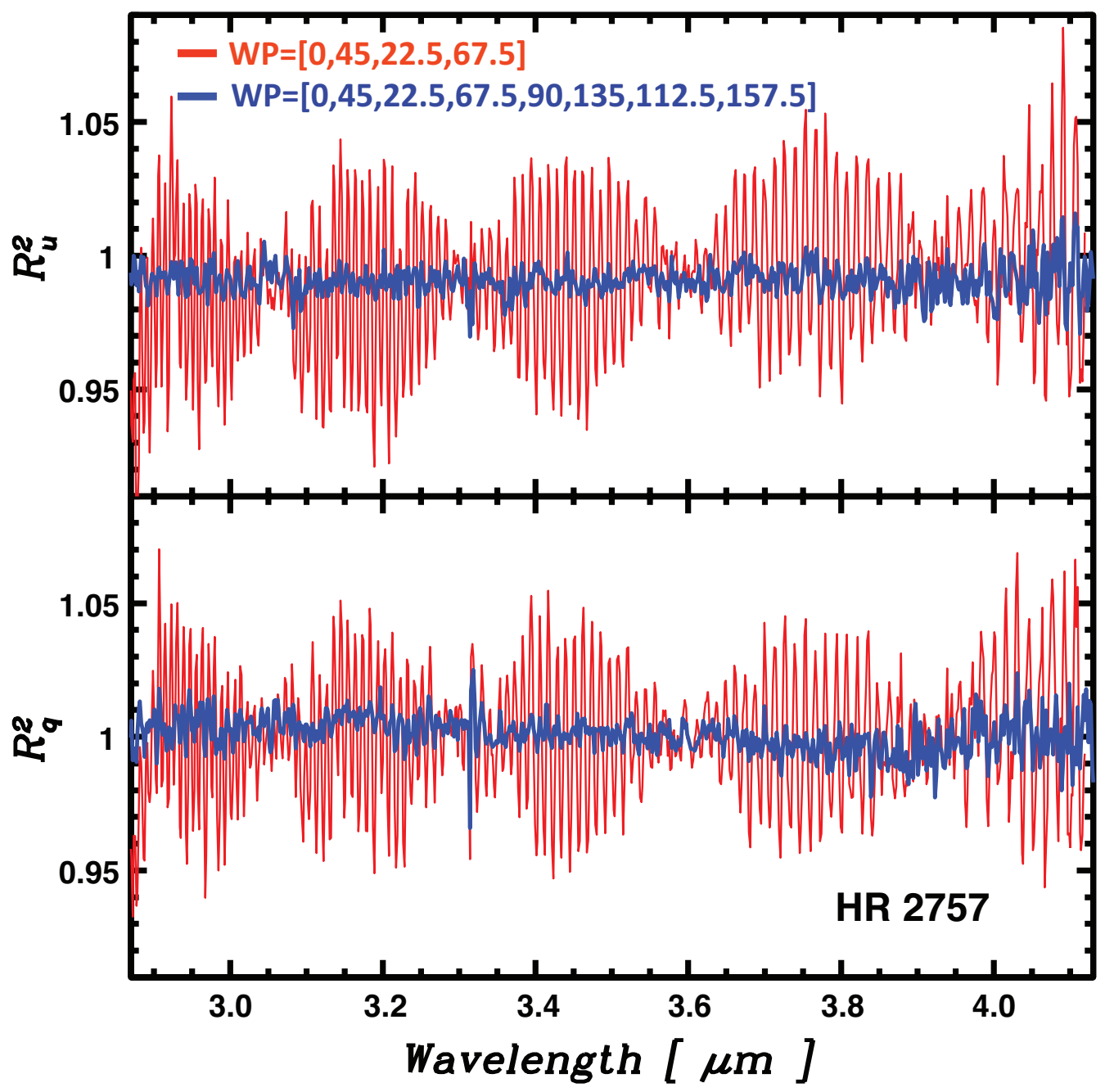

Figure 8. Demonstration of ripple-free polarimetric spectra. The red line is based on a standard operation of $\theta_{W P}=\left[0^{\circ}, 45^{\circ}, 22.5^{\circ}, 67.5^{\circ}\right]$, where $R_{q}^{2}=\left(I_{\| \mid}\left(0^{\circ}\right) / I_{\perp}\left(0^{\circ}\right)\right) /\left(I_{\| \mid}\left(45^{\circ}\right) / I_{\perp}\left(45^{\circ}\right)\right) \quad$ and $R_{u}^{2}=$ $\left(I_{\| \mid}\left(22.5^{\circ}\right) / I_{\perp}\left(22.5^{\circ}\right)\right) /\left(I_{\|}\left(67.5^{\circ}\right) / I_{\perp}\left(67.5^{\circ}\right)\right)$. In case of the $\theta_{W P}=\left[0^{\circ}, 45^{\circ}, 22.5^{\circ}, 67.5^{\circ}, 90^{\circ}, 135^{\circ}, 112.5^{\circ}, 157.5^{\circ}\right]$ operation, the blue line shows a good cancellation of the ripple feature that is seen in red, where $R_{q}^{2}=\left(I_{\|}\left(0^{\circ}+90^{\circ}\right) / I_{\perp}\left(0^{\circ}+\right.\right.$ $\left.90^{\circ}\right) /\left(I_{\| \mid}\left(45^{\circ}+135^{\circ}\right) / I_{\perp}\left(45^{\circ}+135^{\circ}\right)\right)$ and $R_{u}^{2}=\left(I_{\| \mid}\left(22.5^{\circ}+112.5^{\circ}\right) / I_{\perp}\left(22.5^{\circ}+112.5^{\circ}\right) /\left(I_{\|}\left(67.5^{\circ}+157.5^{\circ}\right) / I_{\perp}\left(67.5^{\circ}+\right.\right.\right.$ $\left.\left.157.5^{\circ}\right)\right)$. 
[4] H. Terada, N. Kobayashi, A. T. Tokunaga, T. S. Pyo, K. Nedachi, M. Weber, R. Potter, and P. M. Onaka, "Performance update of the infrared camera and spectrograph for the Subaru Telescope (IRCS)", in Groundbased Instrumentation for Astronomy, A. F. Moorwood, and M. Iye, eds., Proc. SPIE, 5492, pp. 1542-1550, 2004.

[5] H. Takami, N. Takato, Y. Hayano, M. Iye, S. Oya, Y. Kamata, T. Kanzawa, Y. Minowa, M. Otsubo, K. Nakashima, W. Gaessler, and D. Saint-Jacques, "Performance of Subaru Cassegrain Adaptive Optics System", PASJ, 56, pp. 225-234, 2004.

[6] Y. Hayano, H. Takami, S. Oya, M. Hattori, Y. Saito, M. Watanabe, O. Guyon, Y. Minowa, S. E. Egner, M. Ito, V. Garrel, S. Colley, T. Golota, and M. Iye, "Commissioning status of Subaru laser guide star adaptive optics system", in Adaptive Optics System II, Proc. SPIE, 7736, 77360N, 2010.

[7] M. Watanabe, T. -S. Pyo, H. Terada, T. Hattori, Y. Hayano, Y. Minowa, S. Oya, M. Hattori, T. Kudo, M. Morii, and Jun Hashimoto, "Near-Infrared Adaptive Optics Imaging- and Spectro-polarimetry with the Infrared Camera and Spectrograph of the Subaru Telescope", in Ground-based and Airborne Instrumentation for Astronomy VII, Proc. SPIE, 2018.

[8] J. de Boer, J. H. Girard, D. Mawet, F. Snik, C. U. Keller, and J. Milli, "Characterizing Instrumental Effects on Polarization at a Nasmyth focus using NaCo", in Ground-based and Airborne Instrumentation for Astronomy V., S. K. Ramsay, I. S. McLean, and H. Takami, eds., Proc. SPIE, 9147, pp. 87-101, 2014.

[9] F. Joos, E. Buenzli, H. M. Schmid, and C. Thalmann, "Reduction of polarimetric data using Mueller calculus applied to Nasmyth instruments", in Observatory Operations: Strategies, Processes, and Systems II, R. J. Brissenden, and D. Silva, eds., Proc. SPIE, 7016, pp. 1-11, 2008.

[10] S. K. Leggett, T. G. Hawarden, M. J. Currie, A. J. Adamson, T. C. Carroll, T. H. Kerr, O. P. Kuhn, M. S. Seriger, W. P. Varricatt, and T. Wold, MNRAS, 345, pp. 144-152, 2003.

[11] Gehrels, T. (ed.), "Planets, Stars \& Nebulae studied with Photopolarimetry", Tuscon: University of Arizona Press, pp 168-169, 1974.

[12] D. A. Turnshek, R. C. Bohlin, R. L. Williamson, O. L. Lupie, J. Koornneef, D. H. Morgan, "An atlas of Hubble Space Telescope photometric, spectrophotometric, and polarimetric calibration objects", $A J$, 99, pp. 1243-1261, 1990.

[13] T. J. Jones and D. Klebe, "A simple infrared polarimeter", PASP, 100, pp. 1158-1161, 1988.

[14] P. G. Martin, A. J. Adamson, D. C. B. Whittet, J. H. Hough, J. A.Bailey, S.-H. Kim, S. Sato, M. Tamura, and T. Yamashita, "INTERSTELLAR POLARIZATION FROM 3 TO 5 MICRONS IN REDDENED STARS", ApJ, 392, pp. 691-701, 1992.

[15] R. P. Holloway, A. Chrysostomou, D. K. Aitken, J. H. Hough, and A. McCall, "Spectropolarimetry of the 3- $\mu \mathrm{m}$ water ice feature towards young stellar objects", MNRAS, 336, pp. 425-435, 2002.

[16] S. P. Quanz, H. Avenhaus, E. Buenzli, A. Garufi, H. M. Schmid, and S. Wolf, "Gaps in the HD 169142 Protoplanetary Disk Revealed by Polarimetric Imaging: Signs of Ongoing Planet Formation?", ApJ, 766, pp. L2-L7, 2013.

[17] H. Terada and A. T. Tokunaga, "MULTI-EPOCH DETECTIONS OF WATER ICE ABSORPTION IN EDGE-ON DISKS AROUND HERBIG AE STARS: PDS 144N and PDS 453", ApJ, 834, pp. 115-121, 2017.

[18] K. W. Hodapp, R. Suzuki, M. Tamura, L. Abe, H. Suto, R. Kandori, J. Morino, T. Nishimura, H. Takami, O. Guyon, S. Jacobson, V. Stahlberger, H. Yamada, R. Shelton, J. Hashimoto, A. Tavrov, J. Nishikawa, N. Ukita, H. Izumiura, M. Hayashi, T. Nakajima, T. Yamada, and T. Usuda, "HiCIAO: the Subaru Telescope's new high-contrast coronographic imager for adaptive optics", in Ground-based and Airborne Instrumentation for Astronomy II., I. S. McLean and M. M. Casali, eds., Proc. SPIE, 7014, pp. 19-30, 2008.

[19] S. Hinkley, B. R. Oppenheimer, R. Soummer, D. Brenner, J. R. Graham, M. D. Perrin, A. Sivaramakrishnan, J. P. Lloyd, L. D. Roberts Jr., and J. Kuhn, "SPECKLE SUPPRESSION THROUGH DUAL IMAGING POLARIMETRY, AND A GROUND-BASED IMAGE OF THE HR 4796A CIRCUMSTELLAR DISK", ApJ, 701, pp. 804-810, 2009.

[20] A. J. Adamson and D. C. B. Whittet, "A SEARCH FOR POLARIZATION IN DIFFUSE INTERSTELLAR BANDS TOWARD HD 197770", ApJL, 448, pp. 49-52, 1995.

[21] D. K. Aitken and J. H. Hough, "Spectral Modulation, or Ripple, in Retardation Plates for Linear and Circular Polarization", PASP, 113, 1300-1305, 2001. 
Table 2. Observing log for TIR polarimetry engineering.

\begin{tabular}{|l|c|c|c|l|l|}
\hline $\begin{array}{l}\text { Object } \\
\text { Name }\end{array}$ & $\begin{array}{c}\text { Date } \\
(\mathrm{UT})\end{array}$ & $\begin{array}{c}\text { Polarimetric } \\
\text { mode/band }\end{array}$ & $\begin{array}{c}\text { Exp. Time } \\
(\mathrm{s})\end{array}$ & Note & Ref. \\
\hline \hline FS 147 & $2016-06-20$ & Imag. $K^{\prime}, L^{\prime}, M^{\prime}$ & $1.648,15,22.5$ & Photmetric std. & {$[10]$} \\
\hline HD 203856 & $2017-05-20$ & Imag. $H_{2} O$ Ice, $L^{\prime}$ & 200,96 & Nearby star & {$[10]$} \\
\hline HD 210027 & $2016-06-20$ & Spec. $L$ & 160 & Unpolarized star & {$[11]$} \\
\hline BD+32 3739 & $2016-06-20$ & Imag. $K^{\prime}, L^{\prime}$, Spec. $K$ & $80,240,240$ & Unpolarized star & {$[12]$} \\
\hline HD 154892 & $2017-05-19$ & Imag. $H_{2} O$ Ice, $L^{\prime}$ & 200,48 & Unpolarized star & {$[12]$} \\
\hline HD 162208 & $2017-05-20$ & Imag. $H_{2} O$ Ice, $L^{\prime}$, Spec. $L$ & $200,48,240$ & Unpolarized star & {$[13]$} \\
\hline Cyg OB2 No.12 & $2016-06-20$ & Imag. $M^{\prime}$, Spec. $L$ & $48,131.84$ & Polarized star & {$[14]$} \\
\hline Cyg OB2 No.3 & $2016-06-20$ & Imag. $K^{\prime}, L^{\prime}$, Spec. $K$ & $48,48,192$ & Polarized star & {$[14]$} \\
\hline HD 150193 & $2017-05-19$ & Imag. $H_{2} O$ Ice, $L^{\prime}(\mathrm{w} / \mathrm{ND})$ & 48,120 & Polarized star & {$[14]$} \\
\hline Elias 2-25 & $2017-05-20$ & Imag. $L^{\prime}$ & 48 & Polarized star & {$[14]$} \\
\hline GL 2136 & $2017-05-19$ & Imag. $L^{\prime}$, Spec. $K, L$ & $20,480,320$ & Polarized star & {$[15]$} \\
\hline HD 169141 & $2017-05-20$ & Imag. $H_{2} O$ Ice & 100 & Herbig Ae star & {$[16]$} \\
\hline PDS 453 & $2017-05-20$ & Spec. $L$ & 1440 & Herbig Ae star & {$[17]$} \\
\hline
\end{tabular}

